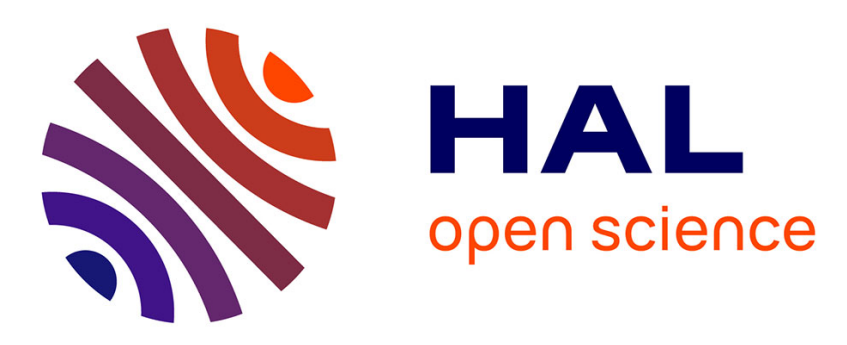

\title{
Credibilist SLAM Performances with Different Laser Set-ups
}

Guillaume Trehard, Evangeline Pollard, Benazouz Bradai, Fawzi Nashashibi

\section{To cite this version:}

Guillaume Trehard, Evangeline Pollard, Benazouz Bradai, Fawzi Nashashibi. Credibilist SLAM Performances with Different Laser Set-ups. ICARCV 2014 - 13th International Conference on Control, Automation, Robotics and Vision, Dec 2014, Singapore, France. hal-01091944

\section{HAL Id: hal-01091944 \\ https://inria.hal.science/hal-01091944}

Submitted on 8 Dec 2014

HAL is a multi-disciplinary open access archive for the deposit and dissemination of scientific research documents, whether they are published or not. The documents may come from teaching and research institutions in France or abroad, or from public or private research centers.
L'archive ouverte pluridisciplinaire HAL, est destinée au dépôt et à la diffusion de documents scientifiques de niveau recherche, publiés ou non, émanant des établissements d'enseignement et de recherche français ou étrangers, des laboratoires publics ou privés. 


\title{
Credibilist SLAM Performances with Different Laser Set-ups
}

\author{
Guillaume Trehard*, Evangeline Pollard*, Benazouz Bradai ${ }^{\dagger}$ and Fawzi Nashashibi* \\ *RITS Team, INRIA \\ Domaine de Voluceau, 78150 Rocquencourt, FRANCE \\ https://team.inria.fr/rits \\ †Valeo Driving Assistance Research France \\ 34, rue Saint Andr, 93012 BOBIGNY Cedex, FRANCE
}

\begin{abstract}
Navigation in the Intelligent Transportation Systems (ITS) domain is still divided between reliable solutions that require heavy and costly set-ups and affordable solutions that still lack performances. By proposing a new method for Simultaneous Localisation and Mapping (SLAM) based on Transferable Belief Model (TBM), the authors aimed at finding a reasonable compromise for urban environment [1]. This article supports this choice and proposes a comparison between different laser set-ups to expose advantages and drawbacks of this solution.
\end{abstract}

\section{INTRODUCTION}

Aiming at proposing a safer and more efficient mobility, Intelligent Transportation Systems (ITS) remain nowadays an important field of research for computer science communities. If major steps forward have been achieved over the last few years with demonstrations such as the DARPA Urban Challenge in 2007 [2], [3] or the VisLab PROUD car test in 2013 [4], locating a vehicle and mapping its environment in a urban context and with a reasonable set of sensors is still challenging.

From the mid eighties [5], [6], Simultaneous Localisation and Mapping (SLAM) solutions have benefited of the probabilistic framework and this problem is now considered to be theoretically solved [7]-[9]. By exploiting data from a LIDAR, a Radar or a Vision set-up, SLAM algorithms use the correlation between the vehicle pose and some static landmarks to estimate both the surrounding map of the mobile robot and its own position in this map.

Historical methods track landmarks from one data scan to another with Bayesian filtering systems to deduce the vehicle displacement [7]-[9]. However, these methods mostly use landmark representations of the environment which requires a dedicated extraction algorithm. Other approaches based on Maximisation Likelihood (ML-SLAM) are mainly based on a grid map representation named occupancy grid [10], [11]. These methods build a grid map of the immediate environment of the vehicle and search for the best match between each data scan and the so built occupancy grid.

The main controversial hypothesis of SLAM algorithms lies in considering these input landmarks as static objects of the environment and then relying on an advantageous proportion between the truly static ones and the others, which are actually mobile. If lots of situations could fall under this assumption without important consequences, urban contexts are different as there can be far too many mobile obstacles to assume a static world.

Solutions to this problem consist in using pre-recorded map to provide a neutral and trustful support [12] or adding Mobile Object Tracking algorithms to the SLAM process (SLAMMOT) [11]. Other localization sensors could also support the SLAM algorithm in order to provide a hybrid navigation system that uses data fusion techniques [13], [14].

The proposition made by the authors in [1] was to use both the properties of Transferable Belief Models (TBM) [15] and the ML-SLAM architecture [10] in order to quantify the belief in a landmark to be static or not. This quantification thus avoids the above hypothesis and so leads to more robust SLAM algorithms in crowded environment.

The TBM framework, designed to deal with the data fusion problematic, indeed enables to explicitly describe conflicts between two sources of information, meaning between a built occupancy grid and a new data scan in the current reference. The conflict situations are generated from incoherences in position of landmarks through the time and so the TBM framework describes potential mobile objects, false alarms or ground impacts. Taking these situations into account then enables to weight the impact of a landmark in the SLAM process.

This article aims at validating this Credibilist SLAM and proposing a comparison of its performances between four classical LIDAR set-ups. A first part introduces the TBM framework and its application to occupancy grid, a second part overviews the Credibilist SLAM process and a last part compares the above mentioned set-ups.

\section{CREDibility FRAMEWORK TO REPRESENT LASER DATA}

\section{A. Credibilist Occupancy Grid}

Introduced by Smeth in 1994 [15], the Transferable Belief Model (TBM) leads to a richer representation of knowledge than the probabilistic framework, mainly used in nowadays algorithms, by explicitly characterising the "not known" information. Its adaptation to the grid map framework has been proposed by Moras in [16] to compute a credibilist occupancy grid of the surrounding environment of a vehicle. For each cell of this grid, a Basic Belief Assignment (BBA) is computed according to a given set of exclusive hypotheses 
$\Omega$ and its corresponding power set $2^{\Omega}$.

$$
\begin{gathered}
\Omega=\{\text { Free, Occupied }\} \\
2^{\Omega}=\mathcal{P}(\Omega)=\{A \mid A \subseteq \Omega\}
\end{gathered}
$$

As defined in Eq. (1), the BBA of a cell is thus a set of four masses $m^{\Omega}(A)$ where $A$ represents each singleton such as $A \in 2^{\Omega}=\{$ Free, Occupied, $\Omega, \emptyset\}$.

In the TBM framework, the union Free $\cup$ Occupied describes the lack of knowledge between those two hypotheses (then noted $\Omega$ ) and the element $\emptyset$, called Conflict, represents the part of contradictory information between sources. This contradictory information mainly occurs in case of moving obstacles (detected in a cell and in another one just after), false alarms or ground impacts.

An example of credibilist occupancy grid can be seen in Fig. 1 where the four singletons are color coded and the mass of a singleton $A$ and of a cell $(i, j)$ at time $t$ is noted $m_{i, j, t}^{\Omega}(A)$.

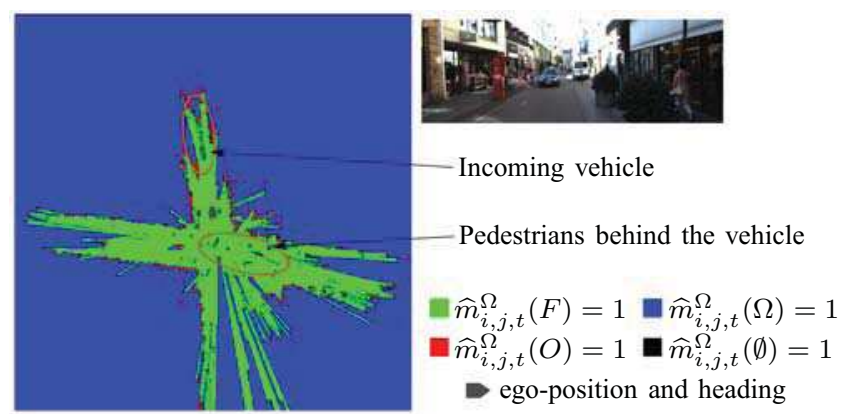

Fig. 1: Example of credibilist occupancy grid and conflict situations

For the rest of the article, the singletons Free and Occupied are respectively denoted by $F$ and $O$.

\section{B. Representation of laser data}

Using the fact that a laser beam provides both the information of an impacted cell and of all the free ones it crossed, a representation has been proposed by Moras in [16]. The same formalism as the one introduced above ( $c f$. Sec. II-A) is used to fill a polar grid map by increasing the Occupied belief of an impacted cell and the Free belief of the crossed ones (Fig. 2).

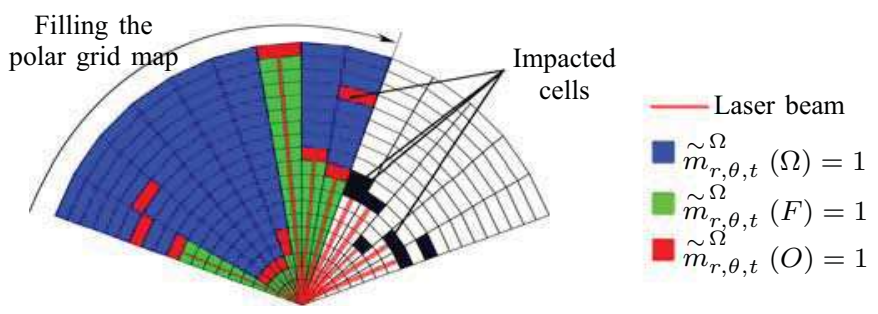

Fig. 2: Filling the polar grid map with a new laser scan

For each cell of the polar grid map, defined by its angle $\theta$ and radius $r$, the measured BBA, denoted $\tilde{m}_{r, \theta, t}^{\Omega}$, is then filled as follows:

$$
\left\{\begin{array}{l}
\tilde{m}_{r, \theta, t}^{\Omega}(A)=\lambda \\
\tilde{m}_{r, \theta, t}^{\Omega}(\Omega)=1-\lambda
\end{array} \quad \text { with } A=\left\{\begin{array}{l}
O \text { if } \text { impacted } \\
F \text { if } \text { crossed }
\end{array}\right.\right.
$$

with $\lambda$ the confidence accorded to the LIDAR sensor.

\section{Normalization with the conflict}

In addition, the proposition has been made in [16] to normalize the occupancy grid with the conflict mass $\widehat{m}_{i, j, t}^{\Omega}(\emptyset)$. This operation has the effect of distributing the belief from the conflict to the other focal elements of the BBA, according to their respective masses. Consequently, the focal element which gathers the highest belief has its mass increased.

$$
\widehat{m}_{i, j, t}^{\Omega}(A)= \begin{cases}\frac{\widehat{m}_{i, j, t}^{\Omega}(A)}{1-\widehat{m}_{i, j, t}^{\Omega}(\emptyset)}, & \text { if } A \neq \emptyset \\ 0, & \text { else }\end{cases}
$$

In other words, if a conflict occurs by updating the map, the hypothesis with the highest belief will be "chosen" as the preference state of the cell.

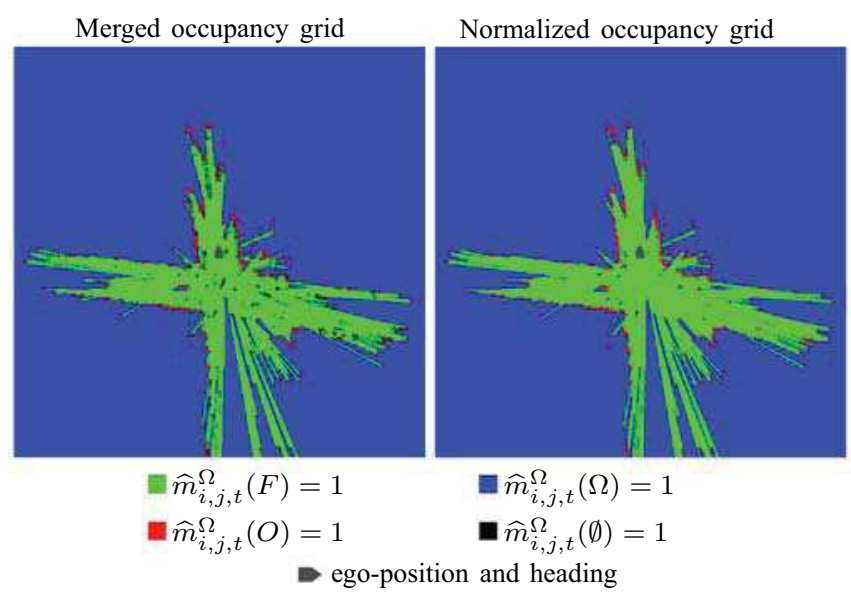

Fig. 3: Effect of normalization by conflict on occupancy grid

Knowing that the conflict is related to mobile objects in the environment or false alarms, the proposition enables to erase such cases and so provides an obstacle free representation of the surrounding area of the vehicle (Fig. 3).

This property of credibilistic occupancy grid is a real advantage when considering Simultaneous Localisation and Mapping (SLAM) algorithms. Where most of probabilistic SLAM techniques indeed assume static their input landmarks without any explicit belief on this hypothesis, the SLAM solution based on the TBM framework uses this belief to weight laser impacts in the SLAM process, and here lays its originality.

\section{CRedibilist SLAM}

The Credibilist SLAM (C-SLAM) concept have been introduced in [1]. It is inspired by a ML-SLAM solution used by Q. Baig et al. [17] and J. Xie et al. [10] and adapted to credibilistic occupancy grid. The main idea is to build at each 
iteration a grid with the on-coming scan and to find the best match between it and the previously recorded grid map (Fig. 4) by using the benefits of the above mentioned property ( $c f$. Sec. II-C) to consider only cells that represent static impacts.

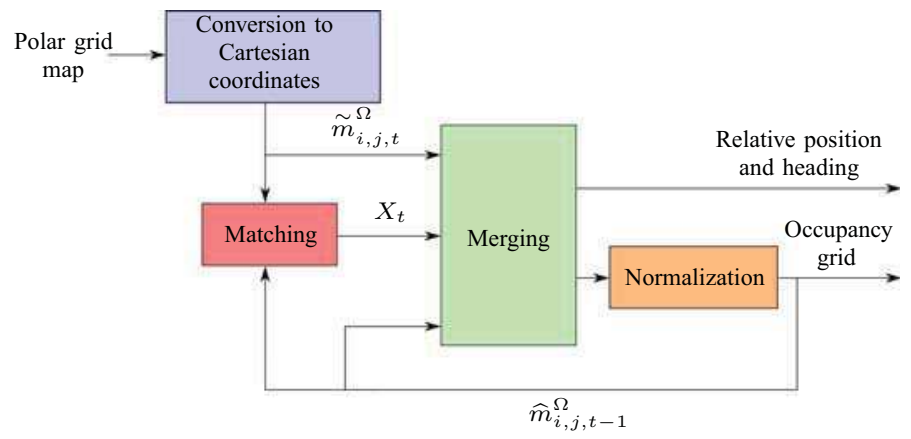

Fig. 4: Overview of the C-SLAM algorithm

The obtained best match corresponds to the vehicle displacement so that both its trajectory and the surrounding map are estimated in the same process. Since the displacement alone leads to a relative localization only, the considered reference in this article is the vehicle reference $R_{0}$ at the beginning of the experiment. It means that at any time $t$, the position of the vehicle $(x, y)$ and its heading $\theta$ are given relatively to the position and the heading at time $t=0$.

\section{A. Matching}

Once the polar grid map has been built and a Cartesian conversion performed, Some candidate states $C$ are computed around an a priori state $\widehat{X}_{t \mid-1}$ based on the previous measurements and according to a basic Constant Speed model.

An operator $O p$ is then applied on each candidate $C$ in order to score the possible corresponding matches between the polar grid map and the stored occupancy grid.

$$
O p\left(\widehat{m}_{i, j, t-1}^{\Omega}, \tilde{m}_{i, j, t}^{\Omega, C}\right)=\sum_{\forall c e l l s} f\left(\widehat{m}_{i, j, t-1}^{\Omega}, \tilde{m}_{i, j, t}^{\Omega, C}\right)
$$

where $\widehat{m}_{i, j, t-1}^{\Omega}$ is the BBA of a cell $(i, j)$ in the occupancy grid reference and $\tilde{m}_{i, j, t}^{\Omega, C}$ is the corresponding BBA in the measured grid map in the candidate $C$ reference. The function $f$ is then defined:

$$
f\left(\widehat{m}_{i, j, t-1}^{\Omega}, \tilde{m}_{i, j, t}^{\Omega, C}\right)=\frac{\left(\widehat{m}_{i, j, t-1}^{\Omega}\left(\text { (U) } \tilde{m}_{i, j, t}^{\Omega, C}\right)(O)\right.}{1-\left(\widehat{m}_{i, j, t-1}^{\Omega}\left(\tilde{m}_{i, j, t}^{\Omega, C}\right)(\emptyset)\right.}
$$

This operator is a sum along all the cells of the polar grid map of a proposed credibilist rule between a cell and its corresponding one in the occupancy grid. It has the effect of favouring the matches between two cells with a heigh Occupied mass and weighting the ones containing a Conflict mass to limit their influence.

The candidate with the highest score is finally chosen as the estimated displacement.

\section{B. Merging}

Considering both the measured grid map and the occupancy grid as reliable sources, Moras et al. [16] propose to use a conjunctive rule to perform the fusion between a new laser scan and the saved map as illustrated in Fig. 5. The candidate selected in the precedent step ( $c f$ Sec. III-A) serves as reference to merge these two sources.

$$
\widehat{m}_{i, j, t}^{\Omega}=\widehat{m}_{i, j, t-1}^{\Omega}(1) \tilde{m}_{i, j, t}^{\Omega}
$$

where $\widehat{m}_{i, j, t-1}^{\Omega}$ is still the BBA of a cell $(i, j)$ in the occupancy grid reference and $\tilde{m}_{i, j, t}^{\Omega}$ is the corresponding BBA in the measured grid map and in the selected candidate reference.

Applied to the SLAM context, this combination leads to estimate the BBA of each cell of the occupancy grid, knowing the previous occupancy grid and the new measured grid map.

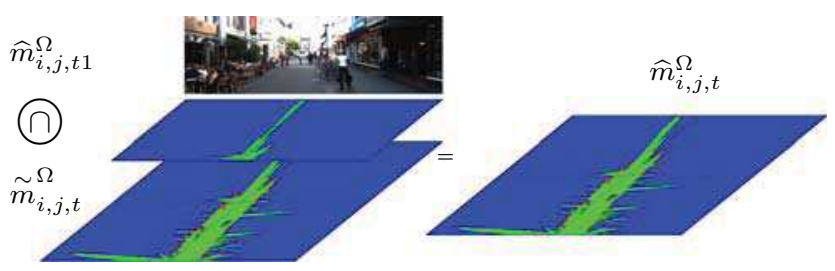

Fig. 5: Merging step of the Credibilist SALM algorithm based on [16]

\section{Results}

Qualitative results are proposed in Fig. 6. They are obtained with a $360^{\circ}$ single layer laser simulated from the KITTI database ( $c f$. Sec. IV-B).

The reader might then find a detailed description of the complete process in the precedent author's work [1].

\section{IMPACT OF LASER SET-UP}

If the matching operator has a major role regarding the performances of the C-SLAM algorithm [1], an important parameter remains the set-up of the lasers on the vehicle. The point cloud generated at each iteration is highly affected by the choice made on the number, the position or the field of view of each embedded LIDAR. The following part of this article aims at quantifying this impact over four classic set-ups:

- Dense 3D laser cloud: Typically the one extracted from a Velodyne sensor. Only ground impacts are ignored with a simple threshold in altitude.

- 360 degrees simple layer: This category could represent a set-up with two LIDARs with a $180^{\circ}$ field of view. One pointing forward; the other one backward.

- 90 degrees back and front: Maybe the better compromise between price and point cloud density, this solution is based on two LIDAR with a field of view of $90^{\circ}$. One is pointing forward, the other one backward.

- 180 degrees front: The most affordable solution. This kind of set-up can be achieved with a simple layer LIDAR, facing forward the vehicle. 

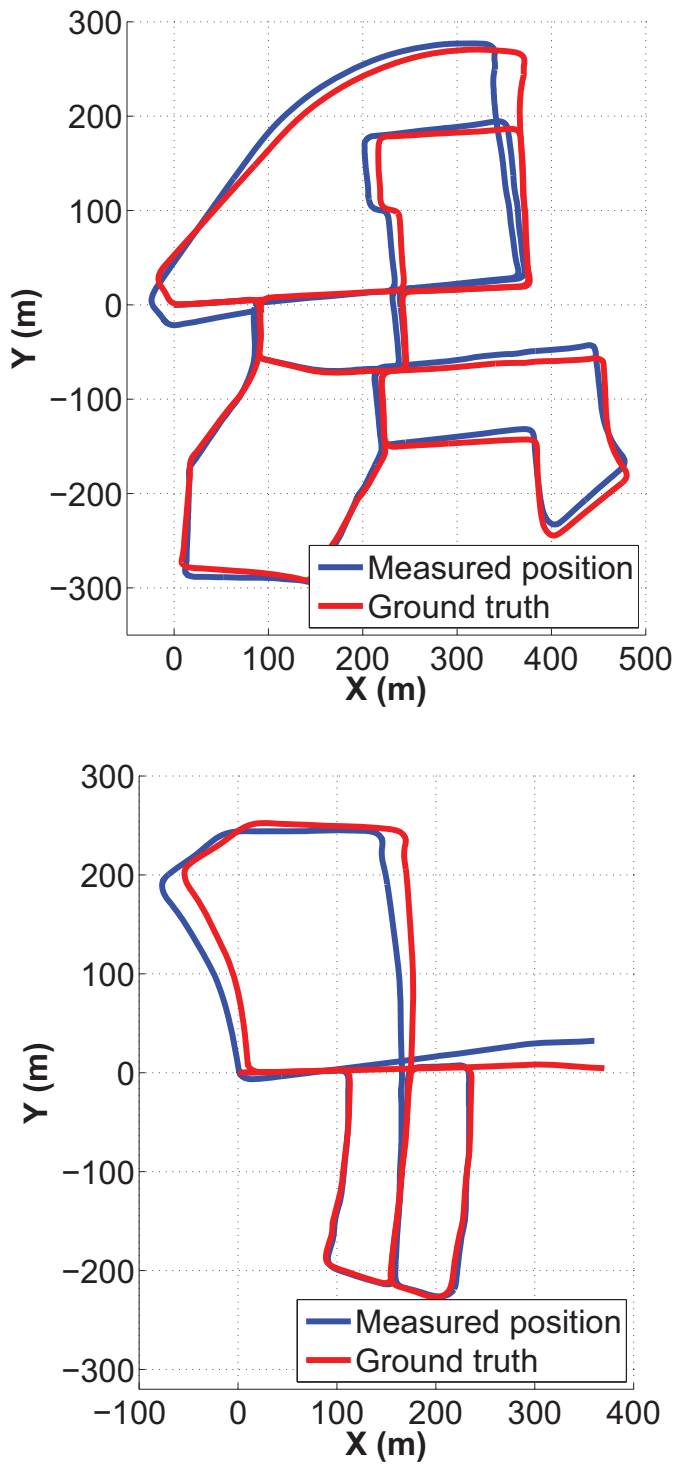

Fig. 6: Example of a results obtained with Credibilist SLAM on sequences from the KITTI database

In order to have qualitative results, all these set-ups are tested with the same grid parameters (resolution of $0.2 \mathrm{~m}$ for the occupancy grid) and evolution model (Constant speed). All the following plotted results follows those parameters too and comparisons are achieved with the same dataset and under the same time references.

\section{A. Operator robustness}

A first interesting result is to compare the scores given in the matching step to candidates around the a priori state ( $c f$. Sec. III-A). These scores indeed allow to chose the displacement by selecting the maximum and it is worth validating this model with poor pieces of information.

Fig. 7 exposes two plots for three of the proposed laser set-ups. The first plot is a 3D representation of the score computed for each candidate in position for the selected angle (the one that leads to the maximum score) and at a random step of one KITTI sequence. The second plot represents the maximum score in position for each computed angle. These plots are representative of the obtained outputs all along the tested sequences.

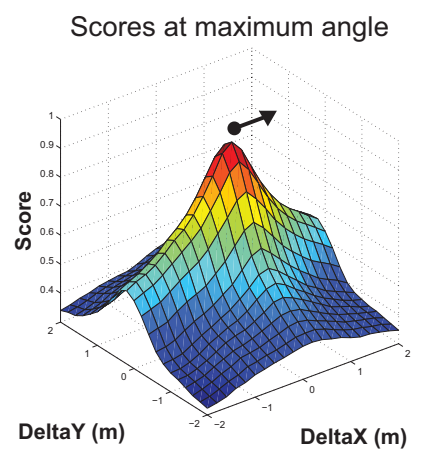

Maximum score along $\mathrm{X}$ and $\mathrm{Y}$ per a

Scores at maximum angle

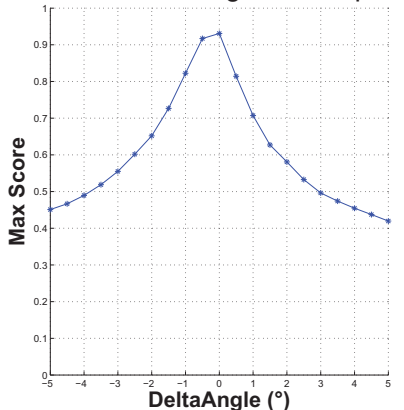

Maximum score along $\mathrm{X}$ and $\mathrm{Y}$ per a

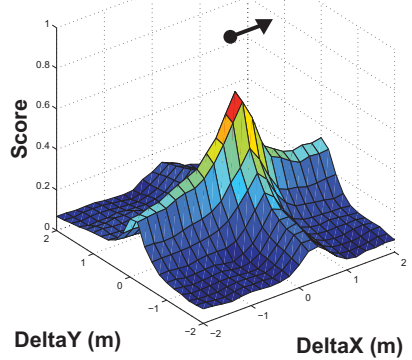

Scores at maximum angle
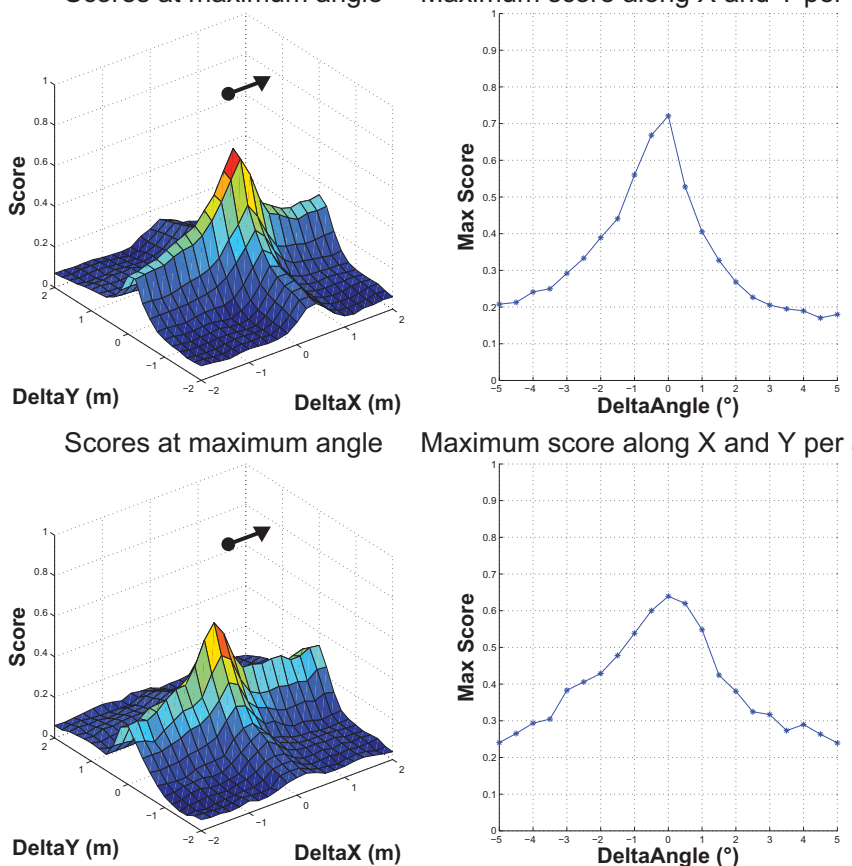

Maximum score along $\mathrm{X}$ and $\mathrm{Y}$ per a

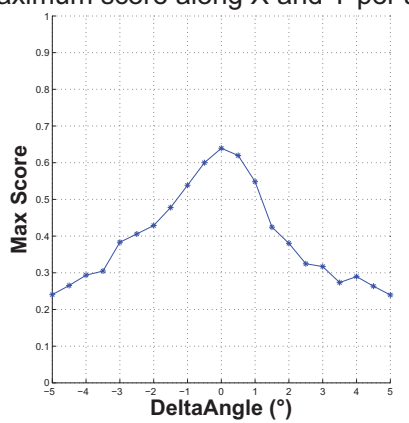

Fig. 7: Examples of output scores at the same step with different Laser layouts

From top to bottom: With a $3 \mathrm{D}$ scan, a $360^{\circ}$ simple layer scan and a $180^{\circ}$ front and simple layer scan

This representation first enables to see that, for the set-up with the maximum of data (the 3D laser cloud), the maximum score search is a convex problem. Hence, the proposed matching operator well serves the purpose because a unique solution can be encountered, at least when a sufficient amount of data is available.

The next results enable to show that, even if the convex hypothesis is not respected for lighter laser set-ups, the scores still seem to present a global maximum which is distinguishable from the local ones around. It is however worth noticing that the difference between higher and lower scores decreases for the two last set-ups. As a consequence, they will be more 
inclined to suffer from urban canyon situations (indecision between several positions in the canyon direction).

\section{B. Set-ups simulation}

Since the simultaneous acquisition of all the solutions on the same path is really hard to achieve, the proposition here is to simulate each of them, based on raw data from the KITTI database [18]. Raw data are taken from a Velodyne HDL-64E placed on the roof of the vehicle, meaning that it covers a $360^{\circ}$ horizontal field of view and a $26.8^{\circ}$ vertical one with a resolution of $0.09^{\circ}$.

The above cited set-ups are then obtained by deteriorating the Velodyne data in terms of field of view and number of layers. Moreover, a lower angular resolution of $0.25^{\circ}$ is used to reach the data quality of affordable LIDAR.

If this method still lacks realism in terms of point of view (the original data are acquired from the roof whereas a basic LIDAR would be placed near bumpers), it enables to have a proper comparison, based on real data, of the performances of the proposed SLAM.

The following results have been obtained on 10 sequences of the odometry dataset proposed by KITTI. They cover a large amount of urban situations and the performances are computed according to the method proposed by the KITTI team in [18].

\section{Position and point density}

In this section a performance comparison is proposed between the four laser set-ups. The results are obtained according to the method introduced in Sec. IV-B and plotted in Fig. 8.

It is first important to see that the 3D cloud set-up performs much better than any other solutions. The credibilist SLAM indeed works in a $2 \mathrm{D}$ context so using projected 3D data as input could have led to instability or errors. Ground impacts and dense point clouds projected on a 2D plane (e.g. from trees...) indeed leads to a large amount of false alarms for the system. However, it appears that it reacts well to this situation and provides benchmark results of $2.04 \%$ in translation errors and $0.0021^{\circ} / \mathrm{m}$ in rotation error. As a comparison, the best score in the KITTI odometry benchmark is obtained with a 3D SLAM using full Velodyne data [19] and reaches $0.76 \%$ in translation error and $0.0020^{\circ} / \mathrm{m}$ in rotation error. Considering the proposed system, reaching such results with such a bad ratio of false alarms confirms the assumption that TBM framework could be worth being used in crowded situations and contexts with lots of moving obstacles.

In addition, all the tests performed with the different set-ups led to robust results (without falls). This adds credits to assume a robust and reliable system in complicated urban environment.

Another interesting result lays in the confrontation between a $180^{\circ}$ front laser and $90^{\circ}$ front and back lasers. Talking about the amount of data, those two set-ups are identical but their performances are actually well distinguishable. The $180^{\circ}$ front laser leads to an average translation error of $19.37 \%$ versus $7.69 \%$ for the $90^{\circ}$ front and back lasers and the average translation errors are respectively equal to $0.0133^{\circ} / \mathrm{m}$ and $0.006^{\circ} / \mathrm{m}$ which differ by more than a factor 2 . This difference
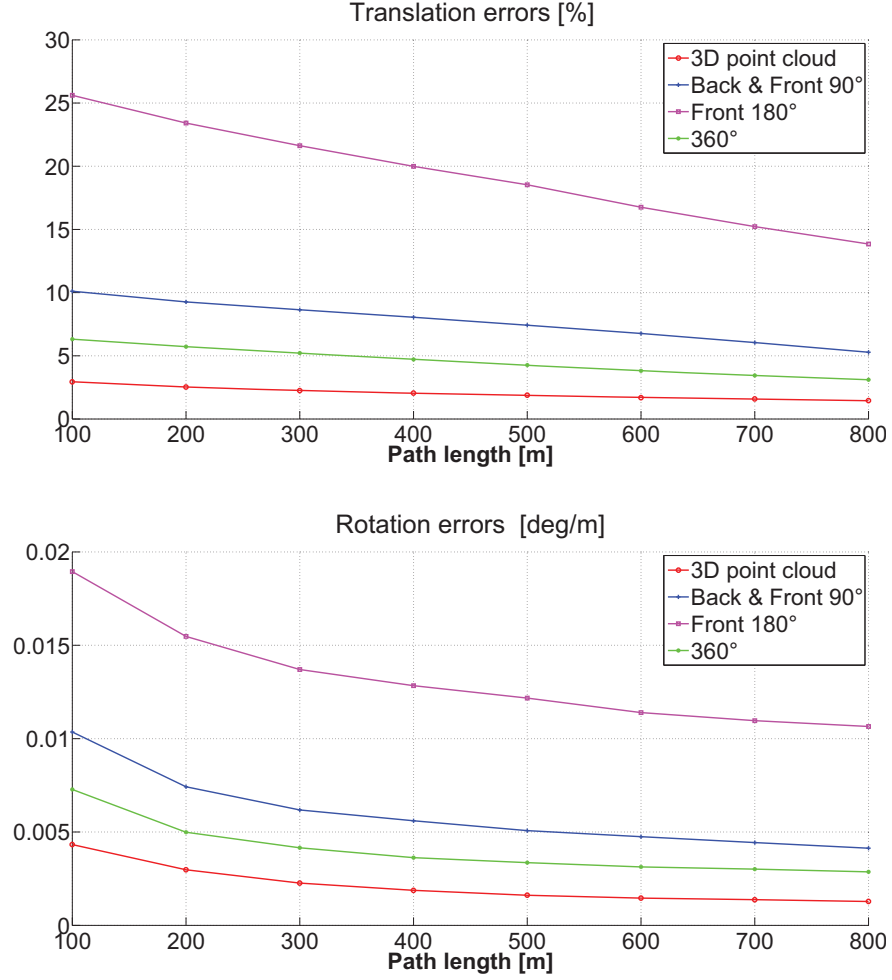

Fig. 8: Translation and rotation errors for 10 sequences of KITTI database

shows the importance, for localization, to have pieces of information from both the front and back of the vehicle. It indeed participates in improving the quality of the orientation estimation and so the whole pose.

It is worth to finally highlight that the $360^{\circ}$ laser set-up lead to average errors of $4.57 \%$ in translation and $0.0041^{\circ} / \mathrm{m}$ in rotation. This configuration could thus afford an interesting compromise in terms of price and performances.

\section{CONCLUSION}

Using the TBM framework along with a ML-SLAM architecture enables to compute and consider the belief in a landmark to be static or not. This belief can then be used in the SLAM process to provide a robust matching even in crowded circumstances.

The proposed matching operator leads to a convex optimisation problem when a sufficient amount of information is available. The method still retains a clear solution when the amount of data is decreasing. This encourages to trust the proposed operator and validate the coherence of the method.

The Credibilist SLAM has demonstrated good performances whatever the laser set-up used. Those set-ups have been benchmarked using the KITTI odometry database. The proposed solution is capable of exploiting the most of a point cloud without being disturbed by false alarms or ground impacts (e.g. the $3 \mathrm{D}$ scan set-up).

However, the proposed set-ups only considered laser sensors and it would be interesting to persue the study by integrating 
GPS and odometry sensors in addition.

Another perspective is to extend the proposed solution to landmark representation context in order to exploit the current existing solutions in this domain.

\section{REFERENCES}

[1] G. Trehard, Z. Alsayed, E. Pollard, B. Bradai, and F. Nashashibi, "Credibilist Simultaneous Localization and Mapping with a LIDAR," in International Conference on Intelligent Robots and Systems (IROS), 2014.

[2] M. Montemerlo, J. Becker, S. Bhat, H. Dahlkamp, D. Dolgov, S. Ettinger, D. Haehnel, T. Hilden, G. Hoffmann, B. Huhnke, D. Johnston, S. Klumpp, D. Langer, A. Levandowski, J. Levinson, J. Marcil, D. Orenstein, J. Paefgen, I. Penny, A. Petrovskaya, M. Pflueger, G. Stanek, D. Stavens, A. Vogt, and S. Thrun, "Junior : The Stanford Entry in the Urban Challenge," Journal of Field Robotics, vol. 25, no. 9, pp. 569-597, 2008.

[3] C. Urmson, J. Anhalt, D. Bagnell, C. Baker, R. Bittner, M. N. Clark, J. Dolan, D. Duggins, T. Galatali, C. Geyer, M. Gittleman, S. Harbaugh, M. Hebert, T. M. Howard, S. Kolski, A. Kelly, M. Likhachev, M. McNaughton, N. Miller, K. Peterson, B. Pilnick, R. Rajkumar, P. Rybski, B. Salesky, Y.-W. Seo, S. Singh, J. Snider, A. Stentz, W. R. Whittaker, Z. Wolkowicki, J. Ziglar, H. Bae, T. Brown, D. Demitrish, B. Litkouhi, J. Nickolaou, V. Sadekar, W. Zhang, J. Struble, M. Taylor, M. Darms, and D. Ferguson, "Autonomous driving in urban environments: Boss and the Urban Challenge," Journal of Field Robotics, vol. 25, no. 8, pp. 425-466, 2008. [Online]. Available: http://dx.doi.org/10.1002/rob.20255

[4] VisLab, "PROUD-Car Test 2013," 2013. [Online]. Available: http://vislab.it/proud-en/

[5] R. C. Smith and P. Cheeseman, "On the representation and estimation of spatial uncertainty," The international journal of Robotics Research, vol. 5 , no. 4 , pp. 56-68, 1986.

[6] J. J. Leonard and H. F. Durrant-Whyte, "Mobile robot localization by tracking geometric beacons," IEEE Transactions on Robotics and Automation, vol. 7, no. 3, pp. 376-382, 1991.

[7] H. F. Durrant-Whyte and T. Bailey, "Simultaneous localization and mapping: part I," Robotics \& Automation Magazine, IEEE, vol. 13, no. 2, pp. 99-110, 2006.

[8] T. Bailey and H. F. Durrant-Whyte, "Simultaneous localization and mapping (SLAM): Part II," Robotics \& Automation Magazine, IEEE, vol. 13 , no. 3 , pp. $108-117,2006$.

[9] S. Thrun, "Robotic mapping: A survey," in Exploring artificial intelligence in the new millennium. Morgan Kaufmann San Mateo, CA, 2002, pp. 1-35.

[10] J. Xie, F. Nashashibi, M. Parent, and O. Garcia-Favrot, "A real-time robust SLAM for large-scale outdoor environments," ITS World Congress, 2010

[11] T.-D. Vu, "Vehicle perception: Localization, mapping with detection, classification and tracking of moving objects," Ph.D. dissertation, Institut National Polytechnique de Grenoble (INPG), 2009.

[12] J. Levinson, M. Montemerlo, and S. Thrun, "Map-based precision vehicle localization in urban environments," in Proceedings of the Robotics: Science and Systems Conference. Citeseer, 2007.

[13] J. Carlson, "Mapping Large, Urban Environments with GPS-Aided SLAM," Ph.D. dissertation, Carnegie Mellon University, 2010.

[14] I. Abuhadrous, "Système embarqué temps réel de localisation et de modélisation 3D par fusion multi-capteur," Ph.D. dissertation, Mines Paristech, 2005

[15] P. Smets and R. Kennes, "The transferable belief model," Artificial intelligence, vol. 66, no. 2, pp. 191-234, 1994.

[16] J. Moras, V. Cherfaoui, and P. Bonnifait, "Credibilist occupancy grids for vehicle perception in dynamic environments," IEEE International Conference on Robotics and Automation (ICRA 2011), pp. 84-89, 2011.

[17] Q. Baig, T.-D. Vu, and O. Aycard, "Online localization and mapping with moving objects detection in dynamic outdoor environments," in IEEE 5th International Conference on Intelligent Computer Communication and Processing, 2009. ICCP 2009. IEEE, 2009, pp. 401-408.

[18] A. Geiger, P. Lenz, and R. Urtasun, "Are we ready for autonomous driving? The KITTI vision benchmark suite," IEEE Conference on Computer Vision and Pattern Recognition (CVPR 2012), pp. 3354-3361, 2012.
[19] J. Zhang and S. Sanjiv, "Lidar Odometry and Mapping in Real-time," in Submitted Paper, 2014. 